\title{
Outcome of 313 Czech Patients With IgA Nephropathy After Renal Transplantation
}

\author{
Dita Maixnerova ${ }^{1 *}$, Petra Hruba ${ }^{2}$, Michaela Neprasova ${ }^{1}$, Kamila Bednarova $^{2}$, \\ Janka Slatinska ${ }^{2}$, Miloslav Suchanek ${ }^{3}$, Marek Kollar $^{4}$, Jan Novak ${ }^{5}$, \\ Vladimir Tesar ${ }^{1}$ and Ondrej Viklicky ${ }^{2}$
}

${ }^{1}$ Department of Nephrology, General Teaching Hospital, 1 st Faculty of Medicine, Prague, Czech Republic, ${ }^{2}$ Department of Nephrology, Institute of Clinical and Experimental Medicine, Prague, Czech Republic, ${ }^{3}$ University of Jan Evangelista Purkyně in Ústí nad Labem, Ústí nad Labem, Czech Republic, ${ }^{4}$ Department of Pathology, Institute of Clinical and Experimental Medicine, Prague, Czech Republic, ${ }^{5}$ Department of Microbiology, University of Alabama at Birmingham, Birmingham, AL, United States

OPEN ACCESS

Edited by:

Wai Lim,

University of Western Australia, Australia

Reviewed by: Jonathan Barratt, University of Leicester, United Kingdom Qing Ge,

Peking University, China

${ }^{*}$ Correspondence:

Dita Maixnerova

ditama@centrum.cz

Specialty section:

This article was submitted to Alloimmunity and Transplantation,

a section of the journal

Frontiers in Immunology

Received: 16 June 2021 Accepted: 03 September 2021 Published: 30 September 2021

Citation:

Maixnerova D, Hruba P, Neprasova M, Bednarova K, Slatinska J, Suchanek M, Kollar M, Novak J, Tesar V and Viklicky O (2021)

Outcome of 313 Czech Patients

With IgA Nephropathy After Renal Transplantation

Front. Immunol. 12:726215. doi: 10.3389/fimmu.2021.726215
The recurrence of IgA nephropathy (IgAN) after kidney transplantation occurs in 20-35\% of patients. The main aim of this study is to evaluate risk factors affecting the course of IgAN after renal biopsy of native kidney and kidney transplant. We evaluated clinical parameters and histological findings at the time of biopsy of native kidney and after kidney transplantation in 313 patients with IgAN with a follow-up of up to 36 years. Using hierarchical clustering method, patients with graft failure $(n=50)$ were divided into two groups based on the mean time from kidney transplant to graft failure (11.2 versus 6.1 years). The time-to-graft failure corresponded well to the time from the renal biopsy of native kidney to end-stage renal disease (5.9 versus 0.4 years). Body mass index, proteinuria, microscopic hematuria, histological evaluation of fibrosis, and crescents at the time of renal biopsy of native kidney were the main variables for the differentiation of the two groups. Higher age of kidney-transplant donor, histological recurrence of IgAN, antibody-mediated rejection, and the onset of microscopic hematuria and proteinuria within 1 year after kidney transplant were also associated with worse graft survival in multivariate Cox regression analysis.

Keywords: IgA nephropathy, kidney transplantation, the recurrence of IgA nephropathy, microscopic hematuria, proteinuria, renal failure

\section{INTRODUCTION}

IgA nephropathy (IgAN) is the most common primary glomerulonephritis with potentially serious outcome leading to end-stage renal disease in $30 \%$ to $50 \%$ of patients within 20 to 30 years from diagnosis. The diagnosis of IgAN requires evaluation of a renal biopsy specimen and finding of IgA immunodeposits (1). A multihit pathophysiological process has been proposed for the development of IgAN, starting with the production of galactose-deficient IgAl and the corresponding autotantibodies driving the production of pathogenic immune complexes, with genetic and environmental contributing factors $(2,3)$. Clinical risk factors predicting poor prognosis include time-averaged proteinuria, hypertension, decreased estimated glomerular filtration rate (eGFR) (2, 
4), as well as histological lesions characterized by the Oxford classification (MEST-C score) $(1,5,6)$. The decision for additional treatment options beyond the recommended RAS blockade should respect the chance of inducing clinical remission, reducing proteinuria, hematuria, stabilizing renal function without further decline of GFR, as well as mitigating the risk of adverse events that may occur with, for example, using corticosteroids and other types of immunosuppression. Thus, the consideration of the risks and benefits needs to be made in individual cases. An early initiation of immunosuppressive regimen in patients with IgAN at risk of progression may slow down the progression to end-stage renal disease. Several new treatment options for patients with IgAN are currently under evaluation in clinical trials (7).

Due to the young age and minimal comorbidities, patients with IgAN are optimal candidates for kidney transplantation. However, the recurrence of the original disease in the graft is a known complication in patients with IgAN and an often cause in the decline of graft function. Other glomerular or vascular lesions secondary to acute or chronic graft rejections might also negatively affect the graft function.

To better understand the graft function, we evaluated in 313 patients with IgAN who received kidney transplant clinical parameters and histological findings at the time of biopsy of native kidney performed in 1991-2013 and after receiving the kidney transplant in 1996-2017. The main aim of our study is to assess the outcome of renal transplants in this cohort. We identified risk factors for disease progression after kidney transplantation and assessed the correlation with the previous rate of decline of renal function in the native kidneys.

\section{MATERIALS AND METHODS}

Our study is a retrospective, multicenter, observational study of patients with biopsy-confirmed IgAN who received kidney transplant at the Institute for Clinical and Experimental Medicine, Prague, Czech Republic. We retrieved all patients with biopsy-confirmed IgAN from Czech registry of renal biopsies $(n=2,377)$ performed in 25 hospitals in the Czech Republic between 1991 and 2017, and all patients transplanted between 1996 and 2017 at the Institute for Clinical and Experimental Medicine $(n=523)$ and their overlap resulted in a final cohort of 313 patients.

Clinical parameters (erythrocyturia, proteinuria, serum creatinine, eGFR, blood pressure, BMI) were retrieved at the time of native kidney biopsy. In addition, crescents and fibrosis were evaluated in biopsy with native IgAN. After kidney transplantation, all transplant parameters (including peak PRA, HLA mismatch, cold ischemia time, dialysis vintage, recipient and donor ages, and genders) were extracted from the hospital database. The occurrence of rising proteinuria $(>0.5 \mathrm{~g} / 24 \mathrm{~h})$ and erythrocyturia (>10 erythrocytes/ $1 \mu \mathrm{l}$ of urine sediment) and all kidney-biopsy findings were also included in analyses. Kidneybiopsy findings for each case were evaluated independently by two specialized nephropathologists. Any unclear cases were discussed with an additional nephropathologist to reach a consensus.
Native kidney biopsies were performed in local hospitals indicated due to impaired renal function and/or urinalysis and evaluated centrally by pathologists from the Institute for Clinical and Experimental Medicine. After transplantation, besides indication biopsies, the center standard-of-care 3-month protocol biopsies were performed for all kidney-transplant recipients. Indication biopsies were performed based on observed deterioration of graft function and/or proteinuria. The decision for renal biopsy after kidney transplantation was made by the medical team of at least six nephrologists. Only $8 \%$ (25/313) patients never had allograft biopsy.

The evaluation of patients with performed renal biopsies was approved by the Ethics Committee of the General University Hospital in Prague, Czech Republic (number 1443/11, S-IV). Written informed consent was obtained from all subjects involved in the study who underwent renal biopsy.

\section{Statistical Analyses}

Agglomerative hierarchical clustering was used for the differentiation of primary data (time from renal biopsy to dialysis, from renal biopsy to transplantation, or from transplantation to graft failure) into two groups. Discriminant analysis with transformed (principal) variables was used to predict the membership of the other variables to the group. The verification of membership was confirmed by Mann-Whitney nonparametric test. Logistic regression was used for the validation of the results of the discriminant analysis and for the calculation of ROC curves in the case of two groups. Response type for logistic regression was binary; prediction accuracy was nearly $100 \%$. All calculations were done by the program XLSTAT (www.xlstat.com), Continuous variables were compared by the Mann-Whitney $U$ test and categorical variables by Pearson's chi-squared test. The deathcensored graft survival was evaluated by Kaplan-Meier analysis using the log-rank test. To investigate the significance of each prognostic factor for allograft loss, univariable Cox proportional hazards models were calculated for clinical parameters at the time of native kidney biopsy (age and gender, proteinuria, erythrocyturia, creatinine), and clinical variables related to kidney transplantation (HLA mismatch, cold ischemia, dialysis vintage, donor age and gender, type of donor, repeated transplantation, proteinuria and erythrocyturia, biopsy-proven reIgAn, and ABMR) were assessed in a univariable model. Potential predictors of graft loss were included in the multivariable model based on their significance in the univariable analysis. $P$ values of $<0.05$ were considered significant. Statistical analysis was performed using IBM SPSS Statistics, Version 24 (International Business Machines Corp.) and GraphPad Prism 5, Version 5.03 (GraphPad Software, Inc.).

\section{RESULTS}

We evaluated 313 patients with histological diagnosis of IgAN in native kidney biopsies who later received kidney transplants. Detailed characteristics of the cohort are in Tables $\mathbf{1}$ and 2 . Forty-four of these patients (14\%) exhibited histological recurrence of IgAN in the graft. Except for younger age of patients with IgAN recurrence, no other predictive factors for 
recurrence of IgAN were identified [recipient gender, donor age, donor gender, retransplantation, living donor, peak PRA (panel reactive antibody), HLA mismatch, dialysis vintage (length of time on dialysis), cold ischemia; Table 2]. Ten-year renal survival was substantially reduced in patients with histologically diagnosed recurrence of IgAN compared to patients without recurrent IgAN (Figure 1). In 23 patients with recurrence of IgAN, the grafts failed (52.3\%). The main causes of graft failure were recurrence of $\operatorname{IgAN}(n=17)$, recurrence of $\operatorname{IgAN}$ in combination with ABMR $(n=2)$, with TCMR $(n=3)$, and a mixed rejection $(n=1)$. In patients without histological recurrence, 35 grafts failed (13\%). The main causes of graft loss were rejections (ABMR, $n=11$; TCMR, $n=2$; mixed rejection, $n=2)$, infections $(n=6)$, surgery complication in early posttransplant period $(n=5)$, chronic allograft nephropathy $(n=4)$, and cardiorenal syndrome $(n=2)$. Erythrocyturia was detected in $29(65.9 \%)$ of the 44 patients with recurrent IgAN. Thus, erythrocyturia (>10 erythrocytes/1 $\mu$ l of urine sediment), common in IgAN patients with native kidneys, was not observed in $34 \%$ of patients with recurrent IgAN. Ten-year renal survival was unfavorable in patients with histologically confirmed recurrent IgAN and microscopic hematuria compared to those with recurrent IgAN but without microscopic hematuria (Figure 2).

Of the total of 313 patients, 33 underwent repeat kidney transplantation (Table 1). Graft failure developed in six patients after second kidney transplantation; these patients had shorter time to the appearance of erythrocyturia both after the first and second renal transplantations (Figure 3).

Subsequently, we evaluated 50 patients (16\%) with graft failure after the first kidney transplant. Erythrocyturia occurred in $31(62 \%)$ of these patients. Histologically verified recurrence of IgAN was confirmed in 23 individuals (46\%). According to the hierarchical clustering method, 50 patients were divided into two groups that were compared using Mann-Whitney test (Table 3). Statistical parameters of both groups are shown in Table 4. Logistic regression analysis confirmed the distribution, as shown in an ROC curve (Figure 4). The time from kidney transplant to graft failure (10.0 versus 5.5 years) correlated well with the distribution of the two groups based on the time from renal biopsy of native kidney to end-stage renal disease (6.8 versus 1.1 years).

Females were overrepresented in the group with a slower loss of renal function (female to male ratio 0.54 versus 0.22 ). Other risk factors, such as body mass index, proteinuria, microscopic hematuria, and histological evaluation of fibrosis and crescents at the time of renal biopsy of native kidney, were also confirmed, but additional factors, including age, renal parameters, and blood pressure at the onset of renal biopsy of native kidney, did not affect the differentiation into two groups (Table 4).

Higher age of the kidney-transplant donor, histologically verified recurrence of IgAN, antibody-mediated rejection, and the onset of microscopic hematuria and proteinuria within 1 year after kidney transplant were associated with worse graft survival

TABLE 1 | The evaluation of 313 Czech patients with histological diagnosis of IgAN (30 patients with two kidney transplantations, 3 patients with three kidney transplantations).

\begin{tabular}{|c|c|c|c|c|c|}
\hline Patients & Numbers & Period from RB to dialysis (years) ${ }^{a}$ & $\begin{array}{l}\text { Period from RB } \\
\text { to Tx (years) }\end{array}$ & $\begin{array}{l}\text { S-Cr levelat diagnosis } \\
(\mu \mathrm{mol} / \mathrm{L})^{\mathrm{a}}\end{array}$ & $\begin{array}{l}\text { Proteinuriaat diagnosis } \\
\qquad(\mathrm{g} / \text { day })^{\mathrm{a}}\end{array}$ \\
\hline After 1. Tx & 280 & 2.99 & 4.91 & 246 & 2.05 \\
\hline Graft failure after 1. Tx & 50 & 1.33 & 2.42 & 301 & 1.15 \\
\hline Preserved renal function after 1 . Tx & 230 & 3.41 & 5.82 & 230 & 2.35 \\
\hline
\end{tabular}

a Median value

IgAN, IgA nephropathy; Tx, kidney transplantation; RB, renal biopsy; S-Cr level, level of serum creatinine.

TABLE 2 | Patients with histological recurrence of IgAN, significant young recipient's age.

\begin{tabular}{|c|c|c|c|c|}
\hline & \multirow{2}{*}{$\begin{array}{c}\text { Total cohort } \\
n=313\end{array}$} & \multicolumn{3}{|c|}{ Histological recurrence of IgAN } \\
\hline & & yes $(n=44)$ & no $(n=269)$ & $p$ value \\
\hline Recipient age, years [range] & $46[20,79]$ & $38[20,62]$ & $47[22,79]$ & $<0.0001$ \\
\hline Recipient gender, male, n (\%) & $253(81 \%)$ & 35 (80\%) & $218(81 \%)$ & 0.837 \\
\hline Donor age, years [range] & $53[6,81]$ & $53[21,73]$ & $53[6,81]$ & 0.588 \\
\hline Donor gender, male, n (\%) & 135 (43\%) & $14(49 \%)$ & $121(36 \%)$ & 0.167 \\
\hline Retransplantation, n (\%) & $33(10.5 \%)$ & $9(21 \%)$ & $24(9 \%)$ & 0.032 \\
\hline Living donor, n (\%) & $90(29 \%)$ & $14(32 \%)$ & $76(28 \%)$ & 0.72 \\
\hline Peak PRA [range] & $4[0,96]$ & $4[0,96]$ & $4[0,92]$ & 0.757 \\
\hline HLA mismatch [range] & $3[0,6]$ & $3[2,6]$ & $3[0,6]$ & 0.856 \\
\hline Dialysis vintage, months [range] & $16[0,97]$ & $16[0,78]$ & $16[0,97]$ & 0.694 \\
\hline Cold ischemia, hours [range] & $13[0,26]$ & $13[0,23]$ & $13[0,26]$ & 0.882 \\
\hline Erythrocyturia post Tx & $74(24 \%)$ & $29(65.9 \%)$ & $45(17.3 \%)$ & $<0.001$ \\
\hline Proteinuria post $\mathrm{Tx}$ & $90(29 \%)$ & $33(75 \%)$ & $57(21.2 \%)$ & $<0.001$ \\
\hline Follow-up, years [range] & $5[0,21]$ & $6[0.5,15]$ & $4[0,21]$ & 0.881 \\
\hline Graft failure n (\%) & $58(18.5 \%)$ & $23(52.3 \%)$ & 35 (13\%) & $<0.001$ \\
\hline Time to graft failure, years [range] & $5[6.3,17.5]$ & $6.9[2.5,15.4]$ & $5.3[0.03,17.5]$ & 0.074 \\
\hline
\end{tabular}


A

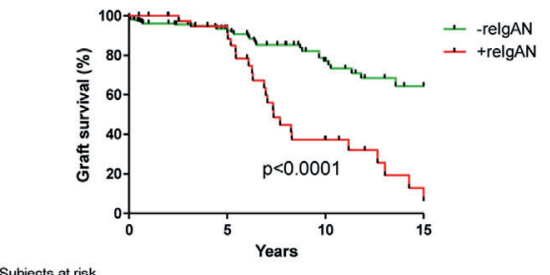

$\begin{array}{lrrrr}\text { Eubjccts at rimk } & & & & \\ \text {-relgAN } & 268 & 126 & 46 & 12 \\ \text { +relgAN } & 44 & 31 & 9 & 2\end{array}$ $1^{\text {st }}$ transplantation

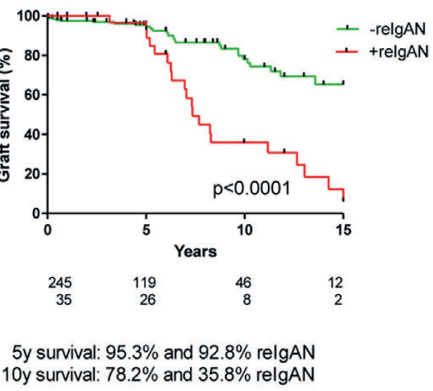

FIGURE 1 | Death-censored graft survival in patients with/without histological recurrence of IgAN in (A) total cohort (log rank $p$ value $p<0.0001)$ and (B) only in recipients who received their first graft (log rank $p$ value $p<0.0001)$.

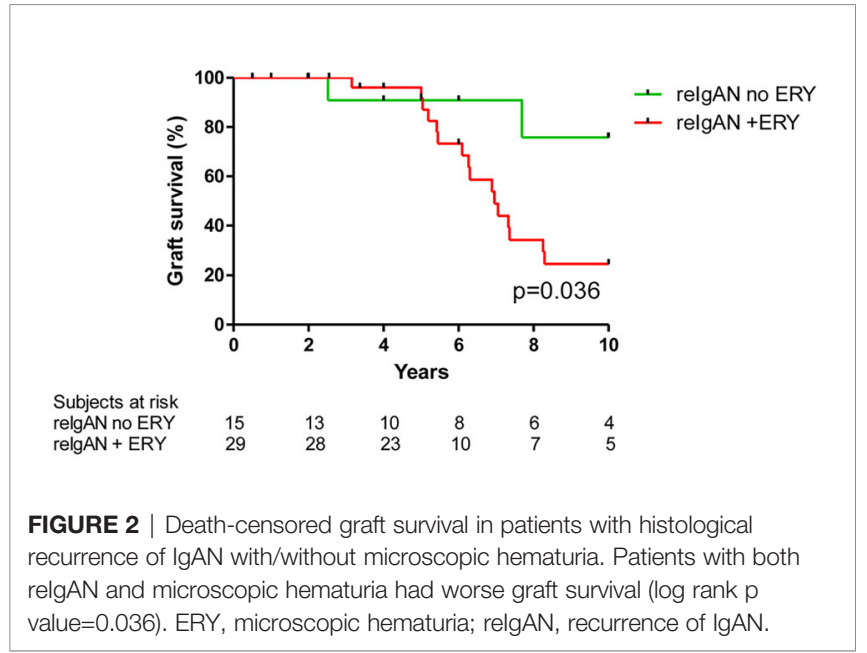

in multivariate Cox regression analysis for patients with original diagnosis of IgAN in native kidney biopsy (Tables 5 and 6).

We did not find differences among subgroups of patients with isolated recurrence of IgAN (i.e., without antibody-mediated rejection), antibody-mediated rejection, or combination of recurrence of IgAN with antibody-mediated rejection on graft survival (Figure 5).

\section{DISCUSSION}

Microscopic hematuria is not a reliable manifestation of IgAN recurrence after kidney transplantation. In an Italian cohort of 190 transplanted patients with IgAN, microscopic hematuria was not present at diagnosis of the recurrence in $25 \%$ of the patients (8), similar to our results showing absence of microscopic hematuria in $34 \%$ of the patients at that time point after

A

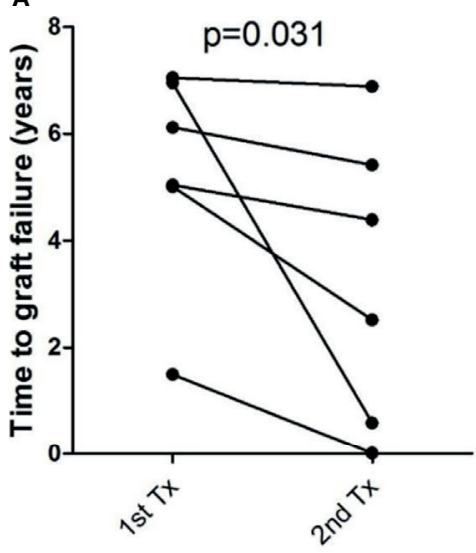

B

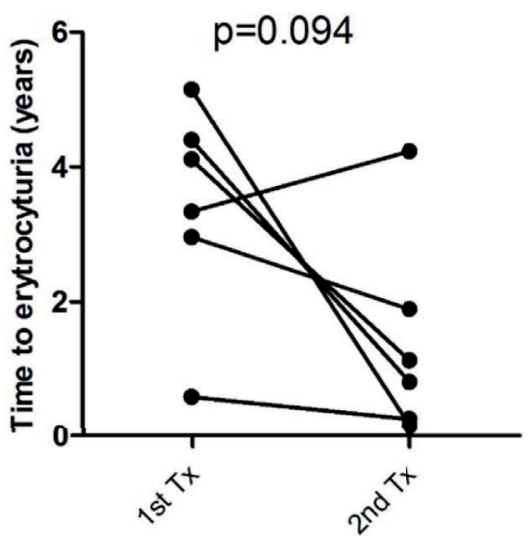

FIGURE 3 | Time to (A) graft failure and (B) erythrocyturia in six patients with repeated transplantation. Patients after second transplantation had shorter time to graft failure $(p=0.031)$ and nonsignificantly shorter time to erythrocyturia $(p=0.094)$. Differences were calculated by Wilcoxon signed rank test. 
TABLE 3 | Two groups of patients with original diagnosis of IgAN after kidney transplant and graft failure were differentiated by agglomerative hierarchical clustering

Statistical parameters

\begin{tabular}{|c|c|c|c|c|c|c|}
\hline \multirow[t]{2}{*}{ Statistic } & \multicolumn{2}{|c|}{ From RB to dialysis (years) } & \multicolumn{2}{|c|}{ From RB to Tx (years) } & \multicolumn{2}{|c|}{ From Tx to graft failure (years) } \\
\hline & group 1 & group 2 & group 1 & group 2 & group 1 & group 2 \\
\hline Nbr. of obs. & 17 & 33 & 17 & 33 & 17 & 33 \\
\hline Median & 5.9 & 0.4 & 7.1 & 2.2 & 11.2 & 6.1 \\
\hline Mean & 6.8 & 1.1 & 6.8 & 2.3 & 10.0 & 5.5 \\
\hline SD & 4.8 & 1.4 & 4.5 & 1.4 & 4.5 & 3.2 \\
\hline Mann-Whitney test & \multicolumn{2}{|c|}{$p<0.0001$} & \multicolumn{2}{|c|}{$p=0.001$} & \multicolumn{2}{|c|}{$p=0.001$} \\
\hline
\end{tabular}

RB, renal biopsy; TX, kidney transplant.

TABLE 4 | Age, gender, renal parameters, proteinuria, erythrocyturia, fibrosis, crescents, age of transplantation, blood pressure, BMl, weight, and height at the time of renal biopsy in two groups of patients with original diagnosis of IgAN after kidney transplant with graft failure.

\begin{tabular}{lccc}
\hline & $\begin{array}{c}\text { Slow } \\
\text { progressors } \\
\text { (group 1) }\end{array}$ & $\begin{array}{c}\text { Rapid } \\
\text { progressors } \\
\text { (group 2) }\end{array}$ & p value \\
\hline $\mathrm{N}$ & 17 & 33 & \\
Age at RB, years & $33.5(9.9)$ & $34.9(12.3)$ & 0.49 \\
Patient gender, male, n (\%) & $11(65 \%)$ & $27(82 \%)$ & $7.8^{\star}$ \\
Creatinine at RB ( $\mu$ mol/L) & $330(184)$ & $399(277)$ & 0.633 \\
eGFR CKD EPI (mL/s) & $0.47(0.36)$ & $0.48(0.46)$ & 0.739 \\
PU at RB (g/24 hours) & $1.52(1.72)$ & $2.93(2.88)$ & 0.077 \\
Erythrocyturia at RB, n (\%) & $15(88 \%)$ & $23(74 \%)^{2}$ & $8.1^{\star}$ \\
Fibrosis at RB, n (\%) & $11(79 \%)^{3}$ & $22(85 \%)^{7}$ & $7.9^{\star}$ \\
Crescents at RB, n (\%) & $4(29 \%)^{3}$ & $17(59 \%)^{4}$ & $6.1^{\star}$ \\
Age of transplantation, & $40(10)$ & $37(12)$ & 0.325 \\
years & & & \\
SPB RB & $155(29)$ & $144(19)$ & 0.285 \\
DPB RB & $99(22)$ & $86(18)$ & 0.208 \\
BMI RB & $24(3)$ & $26(4)$ & 0.033 \\
Weight RB (kg) & $73(10)$ & $78(19)$ & 0.105 \\
Height RB (cm) & $174(11)$ & $171(8)$ & 0.632 \\
\hline
\end{tabular}

${ }^{*}$ Test of proportion; calculated $Z$ value (normal distribution) from transformed binomial distribution; $Z(p=0.05)=1.96$.

Numbers in superscript indicate number of missing values.

eGFR, glomerular filtration rate $(\mathrm{ml} / \mathrm{s}) ; \mathrm{PU}$, proteinuria $(\mathrm{g} /$ day); $S B P$, systolic blood pressure; $D B P$, diastolic blood pressure; $R B$, renal biopsy of native kidney; BMI, body mass index.

Quantitative parameters: mean (SD); qualitative parameters: group frequency (\%).

kidney transplantation. Another study that assessed early recurrence of IgAN in the graft by protocol biopsy showed that $64 \%$ of the patients with recurrent IgAN did not exhibit microscopic hematuria (9). In these patients, histological recurrence of IgAN was found in $1 / 3$ of 65 patients as determined by mesangial IgA immunodeposits in using protocol renal-biopsy specimens with or without mesangial proliferation and with or without mild clinical symptoms (microscopic hematuria and/or proteinuria).

The incidence of IgAN recurrence in biopsies diagnosed based on clinical parameters is approximately $30 \%$, ranging from $9 \%$ to $53 \%$ among different centers with regard to protocol and clinical biopsies (Table 7). This enormous variance might be assigned to diverse biopsy strategies and techniques of histological evaluation in different centers. Thus, the $14 \%$ (44 patients) with histologically verified recurrence of

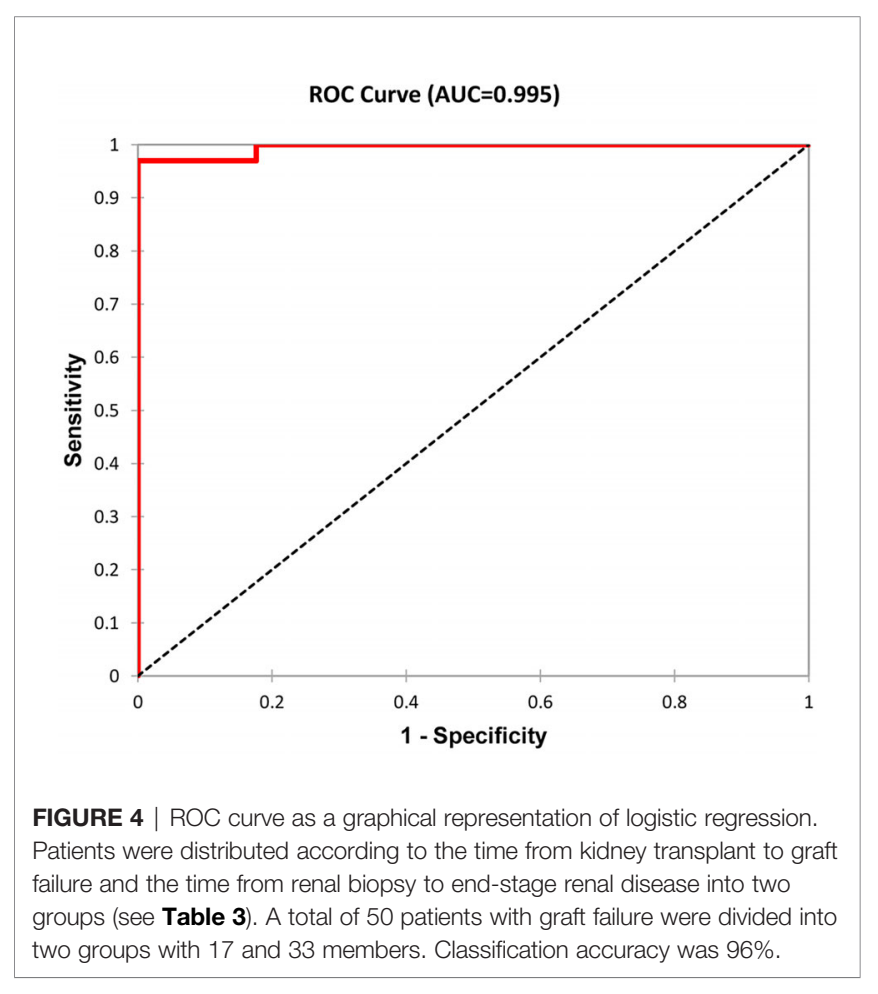

IgAN in our cohort of 313 Czech patients falls just inside this range.

The rate of recurrence after kidney transplant might be undervalued in the absence of a histological diagnosis of the original disease in native kidneys or in the case of short follow-up after kidney transplant (16) and/or in patients who did not undergo the graft biopsy with regard to renal-function decline or proteinuria (17). Additionally, it was assumed that the presence of IgA deposits in donated kidneys (from living or deceased donors) might intensify the possibility of recurrence $(18,19)$. However, when allografts with latent mesangial IgA deposits were transplanted to non-IgAN patients, the IgA immunodeposits were cleared in the recipients, as confirmed by follow-up biopsies (20). Using repeat kidney biopsies, it was shown that in some patients with IgAN, IgA deposits disappear after renal transplantation $(21,22)$. On the other hand, silent IgA deposits might drive clinical IgAN recurrence and disease progression (23). 
TABLE 5 | Analysis of risk factors for graft survival in patients with IgAN in biopsy of native kidney.

\begin{tabular}{|c|c|c|c|c|}
\hline & \multirow[t]{2}{*}{$P$ value } & \multirow[t]{2}{*}{ HR } & \multicolumn{2}{|c|}{$95 \% \mathrm{Cl}$ for $\mathrm{HR}$} \\
\hline & & & Lower & Upper \\
\hline EU till 1 year after Tx & 0.000 & 4.638 & 2.562 & 8.394 \\
\hline PU till 1 year after $T x$ & 0.000 & 7.236 & 3.315 & 15.797 \\
\hline relgAN & 0.000 & 3.305 & 1.937 & 5.640 \\
\hline ABMR & 0.001 & 3.242 & 1.653 & 6.358 \\
\hline Age-recipient & 0.001 & 0.961 & 0.939 & 0.984 \\
\hline Age-RB & 0.002 & 0.962 & 0.939 & 0.986 \\
\hline Retransplantation & 0.060 & 2.167 & 0.967 & 4.855 \\
\hline Recurrence to $2 y$ after Tx & 0.106 & 2.214 & 0.844 & 5.806 \\
\hline Gender-donor, male & 0.112 & 0.606 & 0.326 & 1.124 \\
\hline Age-donor & 0.145 & 1.018 & 0.994 & 1.042 \\
\hline S-Cr at RB & 0.189 & 1.001 & 1.000 & 1.002 \\
\hline PRA max & 0.220 & 0.992 & 0.979 & 1.005 \\
\hline EU native kidney & 0.471 & 0.777 & 0.390 & 1.545 \\
\hline Mismatch & 0.551 & 1.078 & 0.842 & 1.381 \\
\hline CIT hours & 0.626 & 1.009 & 0.973 & 1.047 \\
\hline Gender-recipient, male & 0.686 & 1.145 & 0.593 & 2.212 \\
\hline Dialysis (months) & 0.788 & 1.002 & 0.986 & 1.018 \\
\hline PU at RB & 0.857 & 0.990 & 0.888 & 1.104 \\
\hline Type of donor deceased & 0.975 & 1.009 & 0.577 & 1.764 \\
\hline
\end{tabular}

relgAN, recurrence of IgAN histologically verified; $R B$, renal biopsy; $E S R D$, end-stage renal disease; Tx, kidney transplant; EU, microscopic hematuria; PU, proteinuria; CIT, time of cold ischemia; S-Cr, the level of serum creatinine; ABMR, antibody mediated reaction; $2 y$, two years.

Cox regression model univariate.

TABLE 6 | Multivariate Cox regression model for graft survival.

\begin{tabular}{lcccc}
\hline & Sig. & HR(Exp B) & \multicolumn{2}{c}{$\mathbf{9 5 . 0 \%}$ Cl for Exp (B) } \\
\cline { 4 - 5 } & & & Lower & Upper \\
\hline Donor's age & $\mathbf{0 . 0 4 3}$ & 1.027 & 1.001 & 1.054 \\
Recipient's age & 0.258 & 0.983 & 0.954 & 1.013 \\
relgAN & $\mathbf{0 . 0 2 3}$ & 2.426 & 1.129 & 5.216 \\
ABMR & $\mathbf{0 . 0 0 0}$ & 5.503 & 2.359 & 12.837 \\
EU till 1 year after Tx & $\mathbf{0 . 0 0 8}$ & 3.119 & 1.341 & 7.251 \\
PU till 1 year after Tx & $\mathbf{0 . 0 4 9}$ & 2.791 & 1.003 & 7.767 \\
\hline
\end{tabular}

relgAN, the recurrence of IgAN histological verified; $A B M R$, antibody mediated rejection; EU, microscopic hematuria till 1 year after kidney transplant; PU, proteinuria till 1 year after kidney transplant.

Graft survival in patients with original diagnose of IgAN was influenced by histological verified ABMR ( $H R=5.5, p<0.0001)$, recurrence of IgAN $(H R=2.4, p=0.023)$, the onset of erythrocyturia till 1 year after kidney transplant ( $H R=3.1, p=0.008)$, the onset of proteinuria till 1 year after kidney transplant ( $H R=2.8, p=0.049)$, and higher age of donor $(H R=1.03, p=0.043)$.

The conversion of the histological recurrence into clinical recurrence might be associated with the activation of complement and cytokines production (24). Nevertheless, precise clinical significance of the histological recurrence as well as its effective treatment is unknown; therefore, protocol biopsies in patients with IgAN after kidney transplantation are not recommended. The recurrence of IgAN is a time-dependent event that requires a long follow-up to reach a higher probability of recurrence $(8,10)$.

In our cohort of 313 patients, 10-year renal survival was unfavorable in patients with histologically recurrent IgAN compared to patients without recurrent IgAN (Figure 3). Histologically verified recurrence of IgAN was confirmed in 23

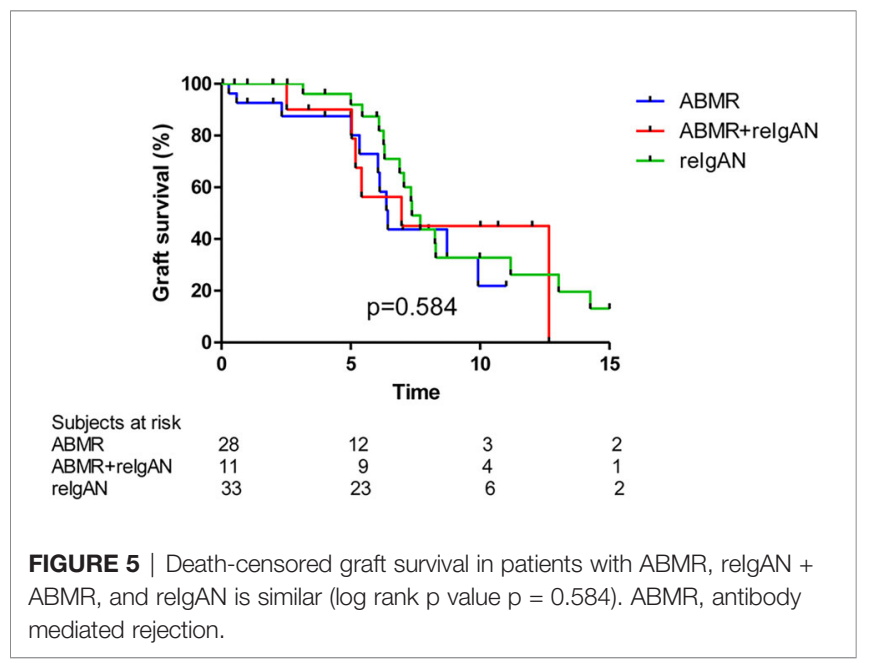

individuals (46\%) of the total number of $50(16 \%)$ patients with IgAN with graft failure in our study, which was a higher percentage compared to the literature (25). The rate of graft loss due to recurrence was reported to range from $1.3 \%$ (46) to $17 \%$ (25). Needless to say, other factors such as glomerular or vascular lesions secondary to acute, chronic graft rejections, or the toxicity of calcineurin inhibitors might influence a graft loss, especially in case of longer time of graft biopsy to the graft function decline (26).

Which predictors of graft failure due to IgAN recurrence in kidney transplant are known? In a cohort of 71 transplanted IgAN patients, cellular or fibrocellular crescents were confirmed in $14 \%$ of graft biopsies and were related to higher interstitial inflammation with a significantly worse graft survival (27). The Oxford classification of histological features of IgAN in the native kidney (28) used for biopsies of recurrent IgAN should have prognostic importance for graft failure $(29,30)$, and, thus, Oxford classification score is suggested to be used for all cases of IgAN recurrence.

The crescents in histological evaluation of native biopsies were an unfavorable factor for renal function of native kidney and for graft survival in our cohort of 313 Czech patients. This finding is in agreement with the results of Columbia University Medical Center from 2001 to 2012 (13). On the other hand, young age at renal transplantation as well as at the onset of IgAN and male gender at renal transplantation reported by others (13, $17,31,32$ ) were not confirmed as risk factors for loss of renal function in our study. Other factors related to kidney-transplant survival were not confirmed in our cohort.

We confirmed the expected clinical risk factors predicting poor outcome in patients with native IgAN, such as proteinuria, erythrocyturia, and body mass index (Table 4). The relation between the body mass index and the probability of end-stage renal disease was shown in Chinese patients with IgAN (33). An increased body mass index associates with lower remission of proteinuria subsequent to treatment in a Japanese cohort (34). It was assumed that obesity increased proteinuria in connection with hypertension and metabolic syndrome. Moreover, the advantages of losing weight in overweight patients with IgAN with protein and sodium restriction and maximal control of 
TABLE 7 | The recurrence of IgAN in kidney transplant.

\begin{tabular}{|c|c|c|c|c|c|c|}
\hline First author & Year of publication & Number of pts & relgAN rate & Time to recurrence (years) & Graft losses & FU \\
\hline Jiang et al. (10) & 2018 & 2393 & 9.9 & 4.6 & & 10 \\
\hline Berthoux et al. (11) & 2017 & 96 & 35.4 & 5.8 & 12.3 & 12.4 \\
\hline Garnier et al. (12) & 2018 & 67 & 20.8 & 3.13 & & 5.9 \\
\hline Avasare et al. (13) & 2017 & 62 & 22.5 & 2.75 & & \\
\hline Von Visger & 2014 & 124 & 22 & & & 6.8 \\
\hline Moroni et al. (8) & 2013 & 190 & 22.1 & 3.7 & 6.3 & 9.4 \\
\hline Mousson (14) & 2007 & 42 & 52.4 & & 4.7 & 8.9 \\
\hline Allen et al. (15) & 2017 & 2501 & 40.7 & & & 7.7 \\
\hline
\end{tabular}

pts, patients; relgAN, recurrence of IgAN, graft losses due to recurrence of IgAN; FU, follow-up.

hypertension using treatment with inhibitors of the reninangiotensin system were confirmed (35).

It was suggested that an increased risk of recurrences and graft loss in patients with IgAN with the first graft loss was due to recurrence $(15,36,37)$, which we confirmed in this study. We detected significantly shorter time to erythrocyturia after first and second kidney transplant and shorter time to second kidney transplantation in patients with IgAN (Figure 3). On the other hand, 7,236 patients of the ANZDATA transplant registry did not have an increased risk of recurrence with graft loss in a subsequent graft in patients with IgAN (10).

Do living-donor kidneys have a more frequent risk of recurrence and graft failure compared to the kidneys from deceased donors? In our cohort, histologically verified recurrence of IgAN as well as the rate of graft survival in recipients from living-related donors was similar to that of recipients from deceased donors. Some studies showed an unfavorable effect of living donors on the graft outcome $(2,8$, 38-41), but other studies failed to confirm this finding (42-44).

We would like to emphasize that our study involves a retrospective analysis of a large cohort of IgAN patients after kidney transplant, comprising data for native kidney biopsies and regular protocolar biopsies of grafts from one center, which, until now, was an underreported area of central Europe regarding this theme. The limitation of our study includes the lack of serum or urinary biomarkers for the assessment of the activity of disease at the time of diagnosis or relapse and/or in association with treatment. Serum IgA levels or serum levels of autoantibodies specific for galactose-deficient IgA1 after kidney transplant or native-kidney biopsy were assessed as the risk factor of IgAN recurrence in other studies $(11,12)$. Another limitation of our study was due to protocol biopsies that were indicated 3 months after kidney transplantation, and, thus, patients with clinically silent recurrent IgAN would be missed. The use of more frequent protocol biopsies was not possible in our transplantation center. Another limitation of our study is missing information about immunosuppressive therapy, use of ACE inhibitors/angiotensin receptor blockers, or statins. Our survey was a single-center study; most patients received maintenance immunosuppression therapy consisting of calcineurin inhibitors, mycophenolate mofetil, and corticosteroids. Most of our patients receive ACE inhibitors. Therefore, adding this information to the model would not provide any substantial effect.

\section{CONCLUSION}

Microscopic hematuria with histologically verified recurrence of IgAN predicts an unfavorable renal survival in a cohort of 313 Czech patients with IgAN who received kidney transplant $(\mathrm{p}<0.001)$. Significantly better renal function was found in female graft recipients. We confirmed that higher BMI, male gender, clinical activity (proteinuria, erythrocyturia), and histological findings, including crescents and enlarged interstitial fibrosis, are risk factors for progressive loss of renal function in patients with IgAN. Furthermore, higher age of donor of the kidney predicted worse prognosis of graft survival of patients with IgAN.

\section{DATA AVAILABILITY STATEMENT}

The original contributions presented in the study are included in the article/supplementary material. Further inquiries can be directed to the corresponding author.

\section{ETHICS STATEMENT}

The studies involving human participants were reviewed and approved by the ethics committee of 1st Medical Faculty, Charles University, Prague, General Teaching Hospital, Prague. The patients/participants provided their written informed consent to participate in this study.

\section{AUTHOR CONTRIBUTIONS}

All authors listed have made a substantial, direct, and intellectual contribution to the work and approved it for publication.

\section{FUNDING}

The authors received funding from grants PROGRES Q25/LF1, DRO VFN 64165, IKEM IN 00023001 from the Ministry of Health of the Czech Republic and NanoEnviCz No. LM2015073 from the Ministry of Education Youth and Sports. 


\section{REFERENCES}

1. Cattran DC, Coppo R, Cook HT, Feehally J, Roberts ISD, Troyanov S, et al. The Oxford Classification of IgA Nephropathy: Rationale, Clinicopathological Correlations, and Classification. Kidney Int (2009) 76:534-45. doi: 10.1038/ki.2009.243

2. Wyatt RJ, Julian BA. IgA Nephropathy. N Engl J Med (2013) 368:2402-14. doi: 10.1056/NEJMra1206793

3. Suzuki H, Kiryluk K, Novak J, Moldoveanu Z, Herr AB, Renfrow MB, et al. The Pathophysiology of IgA Nephropathy. J Am Soc Nephrol (2011) 22:1795803. doi: 10.1681/ASN.2011050464

4. Reich HN, Troyanov S, Scholey JW, Cattran DC. Remission of Proteinuria Improves Prognosis in IgA Nephropathy. J Am Soc Nephrol (2007) 18:317783. doi: 10.1681/ASN.2007050526

5. Barbour SJ, Espino-Hernandez G, Reich HN, Coppo R, Roberts ISD, Feehally $\mathrm{J}$, et al. The MEST Score Provides Earlier Risk Prediction in IgA Nephropathy. Kidney Int (2016) 89:167-75. doi: 10.1038/ki.2015.322

6. Haas M, Verhave JC, Liu ZH, Alpers CE, Barratt J, Becker JU, et al. A Multicenter Study of the Predictive Value of Crescents in IgA Nephropathy. J Am Soc Nephrol (2017) 28:691-701. doi: 10.1681/ ASN.2016040433

7. Maixnerova D, Tesar V. Emerging Modes of Treatment of IgA Nephropathy. IJMS (2020) 23:9064. doi: 10.3390/ijms21239064

8. Moroni G, Quaglini S, Gallelli B, Giovanni Banfi G, Montagnino G, Messa P. The Long-Term Outcome of Renal Transplantation of IgA Nephropathy and the Impact of Recurrence on Graft Survival. Nephrol Dial Transplant (2013) 28:1305-14. doi: 10.1093/ndt/gfs472

9. Ortiz F, Gelpi R, Koskinen P, Manonelles A, Raisanen-Sokolowski A, Carrera M, et al. IgA Nephropathy Recurs Early in the Graft When Assessed by Protocol Biopsy. Nephrol Dial Transplant (2012) 27:2553-8. doi: 10.1093/ndt/ gfr664

10. Jiang SH, Kennard AL, Walters GD. Recurrent Glomerulonephritis Following Renal Transplantation and Impact on Graft Survival. BMC Nephrol (2018) 19:344. doi: 10.1186/s12882-018-1135-7

11. Berthoux F, Suzuki H, Mohey H, Maillard N, Mariat C, Novak J, et al. Prognostic Value of Serum Biomarkers of Autoimmunity for Recurrence of IgA Nephropathy After Kidney Transplantation. J Am Soc Nephrol (2017) 28:1943-50. doi: 10.1681/ASN.2016060670

12. Garnier AS, Duveau A, Demiselle J, Croué A, Subra JF, Sayegh J, et al. Early Post-Transplant Serum IgA Level Is Associated With IgA Nephropathy Recurrence After Kidney Transplantation. PloS One (2018) 13:e0196101. doi: 10.1371/journal.pone.0196101

13. Avasare RS, Rosenstiel PE, Zaky ZS, Tsapepas DS, Appel GB, Markowitz GS, et al. Predicting Post-Transplant Recurrence of IgA Nephropathy: The Importance of Crescents. Am J Nephrol (2017) 45:99-106. doi: 10.1159/ 000453081

14. Mousson C, Charon-Barra C, Funes de la Vega M, Tanter Y, Justrabo E, Martin L, et al. Recurrence of IgA Nephropathy With Crescents After Kidney Transplants. Transplant Proc (2007) 39(8):2595-6. doi: 10.1016/ j.transproceed.2007.08.025

15. Allen PJ, Chadban SJ, Craig JC, Lim WH, Allen RDM, Clayton PA, et al. Recurrent Glomerulonephritis After Kidney Transplantation: Risk Factors and Allograft Outcomes. Kidney Int (2017) 92(2):461-9. doi: 10.1016/ j.kint.2017.03.015

16. Moroni G, Belingheri M, Frontini G, Tamborini F, Messa P. Immunoglobulin A Nephropathy. Recurrence After Renal Transplantation. Front Immunol (2019) 19:10-1332. doi: 10.3389/fimmu.2019.01332

17. Briganti EM, Russ GR, McNeil JJ, Atkins RC, Chadban SJ. Risk of Renal Allograft Loss From Recurrent Glomerulonephritis. N Engl J Med (2002) 347:103-9. doi: 10.1056/NEJMoa013036

18. Suzuki K, Honda K, Tanabe K, Toma H, Nihei H, Yamaguchi Y. Incidence of Latent Mesangial IgA Deposition in Renal Allograft Donors in Japan. Kidney Int (2003) 63:2286-94. doi: 10.1046/j.1523-1755.63.6s.2.x

19. Moriyama T, Nitta K, Suzuki K, Honda K, Horita S, Uchida K, et al. Latent IgA Deposition From Donor Kidney Is the Major Risk Factor for Recurrent IgA Nephropathy in Renal Transplantation. Clin Transplant (2005) 19:41-8. doi: 10.1111/j.1399-0012.2005.00403.x
20. Silva FG, Chander P, Pirani CL, Hardy MA. Disappearance of Glomerular Mesangial IgA Deposits After Renal Allograft Transplantation. Transplantation (1982) 33:241-6.

21. Frohnert PP, Donadio J, Velosa JA, Holley KE, Sterioff S. The Fate of Renal Transplants in Patients With IgA Nephropathy. Clin Transplant (1997) 11:127-33.

22. Hotta O, Furuta T, Chiba S, Tomioka S, Taguma Y. Regression of IgA Nephropathy: A Repeat Biopsy Study. Am J Kidney Dis (2002) 39:493-502. doi: 10.1053/ajkd.2002.31399

23. Kessler M, Hiesse C, Hestin D, Mayeux D, Boubenider K, Charpentier B. Recurrence of Immunoglobulin A Nephropathy After Renal Transplantation in the Cyclosporine Era. Am J Kidney Dis (1996) 28:99-104. doi: 10.1016/ S0272-6386(96)90137-7

24. Coppo R, Feehally J, Glassock RJ. IgA Nephropathy at Two Score and One. Kidney Int (2010) 77:181-6. doi: 10.1038/ki.2009.427

25. Odum J, Peh CA, Clarkson AR, Bannister KM, Seymour AE, Gillis D, et al. Recurrent Mesangial IgA Nephritis Following Renal Transplantation. Nephrol Dial Transplant (1994) 9:309-12.

26. Floege J, Grone HJ. Recurrent IgA Nephropathy in the Renal Allograft:Not a Benign Condition. Nephrol Dial Transplant (2013) 28:1070-3. doi: 10.1093/ ndt/gft077

27. Jeong HJ, Kim YS, Kwon KH, Kim SI, Kim MS, Choi KH, et al. Glomerular Crescents Are Responsible for Chronic Graft Dysfunction in Post-Transplant IgA Nephropathy. Pathol Int (2004) 54:837-42. doi: 10.1111/j.14401827.2004.01751.x

28. Working Group of the International IgA Nephropathy Network and the Renal Pathology SocietyRoberts IS, Cook HT, Troyanov S, Alpers CE, Amore A, et al. The Oxford Classification of IgA Nephropathy: Pathology Definitions, Correlations, and Reproducibility. Kidney Int (2009) 76:546-56. doi: 10.1038/ ki.2009.168

29. Trimarchi H, Barratt J, Cattran DC, Cook HT, Coppo R, Haas M, et al. IgAN Classification Working Group of the International IgA Nephropathy Network and the Renal Pathology Society; Conference Participants. Oxford Classification of IgA Nephropathy: An Update From the IgA Nephropathy Classification Working Group. Kidney Int (2017) 91:1014-21. doi: 10.1016/ j.kint.2017.02.003

30. Park S, Go H, Baek CH, Kim YH, Kim YC, Yang SH, et al. Clinical Importance of the Updated Oxford Classification in Allograft IgA Nephropathy. Am J Transplant (2019) 19:2855-64. doi: 10.1111/ ajt. 15400

31. Han SS, Huh W, Park SK, Ahn C, Han JS, Kim S, et al. Impact of Recurrent Disease and Chronic Allograft Nephropathy on the Long-Term Allograft Outcome in Patients With IgA Nephropathy. Transplant Int (2010) 23:16975. doi: $10.1111 / j .1432-2277.2009 .00966 . x$

32. Ponticelli C, Traversi L, Feliciani A, Cesana BM, Banfi G, Tarantino A. Kidney Transplantation in Patients With IgA Mesangial Glomerulonephritis. Kidney Int (2001) 60:1948-54. doi: 10.1046/j.1523-1755.2001.00006.x

33. Ouyang Y, Xie J, Yang M, Zhang X, Ren H, Wang W, et al. Underweight Is an Independent Risk Factor for Renal Function Deterioration in Patients With IgA Nephropathy. PloS One (2016) 11:e0162044. doi: 10.1371/journal. pone. 0162044

34. Shimamoto M, Ohsawa I, Suzuki H, Hisada A, Nagamachi S, Honda D, et al. Impact of Body Mass Index on Progression of IgA Nephropathy Among Japanese Patients. J Clin Lab Anal (2015) 29:353-60. doi: 10.1002/ jcla. 21778

35. Kittiskulnam P, Kanjanabuch T, Tangmanjitjaroen K, Chancharoenthana W, Praditpornsilpa K, Eiam-Ong S. The Beneficial Effects of Weight Reduction in Overweight Patients With Chronic Proteinuric Immunoglobulin a Nephropathy: A Randomized Controlled Trial. J Ren Nutr (2014) 24:200-7. doi: $10.1053 /$ j.jrn.2014.01.016

36. Freese P, Svalander C, Nordén G, Nyberg G. Clinical Risk Factors for Recurrence of IgA Nephropathy. Clin Transplant (1999) 13:313-7. doi: 10.1034/j.1399-0012.1999.130406.x

37. Ohmacht C, Kliem V, Burg M, Nashan B, Schlitt HJ, Brunkhorst R, et al. Recurrent Immunoglobulin a Nephropathy After Renal Transplantation:a Significant Contributor to Graft Loss. Transplantation (1997) 64:1493-6. doi: 10.1097/00007890-199711270-00024 
38. Donadio JV, Grande JP. IgA Nephropathy. N Engl J Med (2002) 347:738-48. doi: 10.1056/NEJMra020109

39. Gharavi AG, Kiryluk K, Choi M, Li Y, Hou P, Xie J, et al. Genome-Wide Association Study Identifies Susceptibility Loci for IgA Nephropathy. Nat Genet (2011) 43:321-7. doi: 10.1038/ng.787

40. Kiryluk K, Novak J, Gharavi AG. Pathogenesis of Immunoglobulin A Nephropathy: Recent Insight From Genetic Studies. Annu Rev Med (2013) 64:339-56. doi: 10.1146/annurev-med-041811-142014

41. Barbour SJ, Reich HN. Risk Stratification of Patients With IgA Nephropathy. Am J Kidney Dis (2012) 59:865-73. doi: 10.1053/ j.ajkd.2012.02.326

42. US Renal Data System (USRDS). Annual Data Report: Atlas of Chronic Kidney Disease and End Stage Renal Disease in the United States. Bethesda, MD: National Institute of Health, National Institute of Diabetes and Digestive and Kidney Diseases (2013).

43. Choy BY, Chan TM, Lo SK, Lo WK, Lai KN. Renal Transplantation in Patients With Primary Immunoglobulin A Nephropathy. Nephrol Dial Transplant (2003) 18:2399-404. doi: 10.1093/ndt/gfg373

44. Moldoveanu Z, Wyatt RJ, Lee J, Tomana M, Julian BA, Mestecky J, et al. Patients With IgA Nephropathy Have Increased Serum GalactoseDeficient IgA1 Levels. Kidney Int (2007) 71:1148-54. doi: 10.1038/ sj.ki.5002185
Conflict of Interest: JN is a co-founder and co-owner of and consultant for Reliant Glycosciences, LLC, and a co-inventor on US patent application 14/ 318,082 (assigned to UAB Research Foundation).

The remaining authors declare that the research was conducted in the absence of any commercial or financial relationships that could be construed as a potential conflict of interest.

Publisher's Note: All claims expressed in this article are solely those of the authors and do not necessarily represent those of their affiliated organizations, or those of the publisher, the editors and the reviewers. Any product that may be evaluated in this article, or claim that may be made by its manufacturer, is not guaranteed or endorsed by the publisher.

Copyright $\odot 2021$ Maixnerova, Hruba, Neprasova, Bednarova, Slatinska, Suchanek, Kollar, Novak, Tesar and Viklicky. This is an open-access article distributed under the terms of the Creative Commons Attribution License (CC BY). The use, distribution or reproduction in other forums is permitted, provided the original author(s) and the copyright owner(s) are credited and that the original publication in this journal is cited, in accordance with accepted academic practice. No use, distribution or reproduction is permitted which does not comply with these terms. 